\title{
Duality for Convex Monoids
}

\author{
Frank Roumen ${ }^{1}$ (D) . Sutanu Roy ${ }^{2}$
}

Received: 7 March 2016 / Accepted: 27 July 2016/ Published online: 10 August 2016

(C) The Author(s) 2016. This article is published with open access at Springerlink.com

\begin{abstract}
Every $\mathrm{C}^{*}$-algebra gives rise to an effect module and a convex space of states, which are connected via Kadison duality. We explore this duality in several examples, where the $\mathrm{C}^{*}$-algebra is equipped with the structure of a finite-dimensional Hopf algebra. When the Hopf algebra is the function algebra or group algebra of a finite group, the resulting state spaces form convex monoids. We will prove that both these convex monoids can be obtained from the other one by taking a coproduct of density matrices on the irreducible representations. We will also show that the same holds for a tensor product of a group and a function algebra.
\end{abstract}

Keywords Quantum group · Hopf algebra · Effect algebra $\cdot$ Convex space $\cdot$ Kadison duality

\section{Introduction}

States and observables of a physical system are connected via dualities between certain categories. There are several dualities that can be used for this connection. Known examples include the Gelfand duality theorem and the Kadison duality theorem. For a system in classical physics, the state space is modeled by a topological space, and the observables are

Frank Roumen

f.roumen@math.ru.nl

Sutanu Roy

sutanu.roy@carleton.ca

1 Inst. for Mathematics, Astrophysics, and Particle Physics (IMAPP), Radboud University Nijmegen, Heyendaalseweg 135, 6525 AJ Nijmegen, The Netherlands

2 School of Mathematics and Statistics, Carleton University, 1125 Colonel By Drive, Ottawa, ON, K1S 5B6, Canada 
given by functions on this space. In this way, the algebra of observables forms a commutative $\mathrm{C}^{*}$-algebra. The celebrated Gelfand theorem states that the category of locally compact Hausdorff spaces is dually equivalent to the category of commutative $\mathrm{C}^{*}$-algebras, and thus it provides an intimate connection between states and observables. A useful special case occurs when the $\mathrm{C}^{*}$-algebras under consideration have a unit. Gelfand duality in this setting states that the category of unital $\mathrm{C}^{*}$-algebras is dually equivalent to the category of compact Hausdorff spaces.

Gelfand duality does not apply to quantum mechanical systems, since their algebra of observables is in general a non-commutative $\mathrm{C}^{*}$-algebra. There is no good non-commutative analogue of Gelfand duality, but there is a duality theorem due to Kadison that can be useful to describe quantum systems. Kadison duality is not based on $\mathrm{C}^{*}$-algebras, but on the unit interval within a unital $\mathrm{C}^{*}$-algebra. This unit interval forms a structure called an effect module, and there is a dual equivalence between a certain category of effect modules and a certain category of convex spaces. The state space of a quantum system forms a convex space and the corresponding effect module contains its observables; hence Kadison duality connects states and observables of quantum systems. It does not directly generalize Gelfand duality, since the unit interval of a $\mathrm{C}^{*}$-algebra contains less information than the $\mathrm{C}^{*}$-algebra itself.

When studying physical systems, one often wants to take the symmetry group of the system into account. In the $\mathrm{C}^{*}$-algebraic picture, this leads to quantum groups. For ordinary Gelfand duality, we use locally compact Hausdorff spaces as state spaces. If we take the symmetry of a system into account, the state space becomes a locally compact group. On the dual side, this gives a coalgebra structure on the $\mathrm{C}^{*}$-algebra, making it into a structure called a quantum group. There is an analogue of the Gelfand duality theorem that takes the symmetry into account. This theorem states that the category of compact (Hausdorff) groups is dually equivalent to the category of commutative compact quantum groups.

Summarizing, there are two dualities involving topological spaces and $\mathrm{C}^{*}$-algebras: one for systems without symmetry, and one for systems with symmetry. Furthermore, Kadison duality relates convex spaces and effect modules for systems without symmetry. In this article we shall will describe a variant of Kadison duality for systems with symmetry. This will lead to a notion of a quantum group whose underlying algebra is an effect module instead of a $\mathrm{C}^{*}$-algebra. Schematically, we wish to complete the diagram on the right:
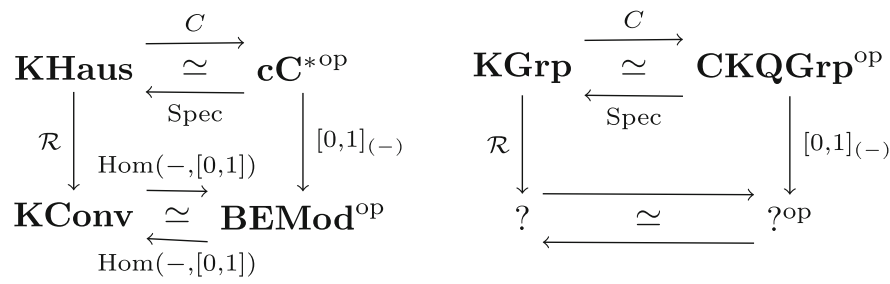

The categories and functors occuring in this diagram will be explained in more detail in the next section. We will restrict our attention to finite groups. In the theory of $\mathrm{C}^{*}$-algebraic quantum groups, there is only one way to assign a commutative quantum group or Hopf-algebra to any finite group. We show that there are two ways to assign an effect module (and a dual convex space) to a finite group, arising from two different Hopf algebras associated to the group. Both ways to form "effect quantum groups" are related via a version of Pontryagin duality. 
The outline of this paper is as follows. Section 2 contains preliminary material about convex spaces, effect modules, and quantum groups. In particular we will describe the various dualities that connect these objects. In Section 3 we will determine the effect modules and convex spaces associated to the group algebra and the function algebra of a finite group. The two convex spaces obtained in this way are both convex monoids, that is, monoids in the category of convex spaces. The connection between these two monoids will be established in Section 4 . We will prove that both convex monoids determine each other via essentially the same construction: if $V_{1}, \ldots, V_{k}$ are the irreducible linear representations of either of these monoids, then the coproduct $\mathcal{D} \mathcal{M}\left(V_{1}\right)+\cdots+\mathcal{D} \mathcal{M}\left(V_{k}\right)$ is a convex monoid isomorphic to the other one. Finally, in Section 5, we will prove a related result for the tensor product of a group and a function algebra.

\section{Preliminaries}

We will present the dualities alluded to in the Introduction in more detail here. The most basic duality that we will use is Gelfand duality. Throughout this paper, we will assume that all $\mathbf{C}^{*}$-algebras we encounter have a unit. Write $\mathbf{C}^{*}$ for the category of $\mathrm{C}^{*}$-algebras with *-homomorphisms as maps. The full subcategory of commutative $\mathrm{C}^{*}$-algebras is denoted cC $^{*}$. Furthermore write KHaus for the category of compact Hausdorff spaces with continuous maps. If $X$ is a compact Hausdorff space, then the collection $C(X)$ of complex-valued functions on $X$ is a commutative $C^{*}$-algebra with pointwise operations. This construction gives a contravariant functor $C$ from KHaus to $\mathbf{c C}^{*}$ by letting it act on morphisms via precomposition. The Gelfand spectrum provides a functor in the other direction: if $A$ is a commutative $C^{*}$-algebra, then its spectrum $\operatorname{Spec} A=\operatorname{Hom}_{\mathbf{c}} C^{*}(A, \mathbb{C})$ is a compact Hausdorff space. The spectrum construction forms a contravariant functor from $\mathbf{c C}^{*}$ to KHaus, again using precomposition.

Theorem 1 (Gelfand) The compositions $C \circ \mathrm{Spec}$ and $\mathrm{Spec} \circ C$ are naturally equivalent to the identity functor. Hence the categories $\mathrm{KHaus}$ and $\mathbf{c C}^{*}$ are dually equivalent.

There is a more general version of Gelfand duality involving non-unital $\mathrm{C}^{*}$-algebras and locally compact spaces, but we will only be concerned with compact spaces in the remainder of this article.

The Gelfand Theorem justifies viewing $\mathrm{C}^{*}$-algebras as a non-commutative generalization of spaces. Similarly it is useful to have a non-commutative generalization of topological groups. This gives the notion of a quantum group. There are several definitions of quantum groups; here we will use the compact quantum groups from Woronowicz [9]. For a general overview of the theory of quantum groups see [8].

Definition 2 A compact quantum group is a $\mathrm{C}^{*}$-algebra $A$ equipped with a unital *-homomorphism $\Delta: A \rightarrow A \otimes A$ called the comultiplication, such that

- The comultiplication is coassociative, i.e. $\left(\Delta \otimes \mathrm{id}_{A}\right) \circ \Delta=\left(\mathrm{id}_{A} \otimes \Delta\right) \circ \Delta$.

- The linear spans of $\Delta(A)(1 \otimes A)$ and $\Delta(A)(A \otimes 1)$ are dense in $A \otimes A$.

If $G$ is a compact Hausdorff group, then its function algebra $C(G)$ is a commutative $\mathrm{C}^{*}$-algebra. It can be made into a compact quantum group by defining $\Delta: C(G) \rightarrow$ 
$C(G) \otimes C(G) \cong C(G \times G), \Delta(\varphi)(g, h)=\varphi(g h)$. This construction provides a grouptheoretic analogue of Gelfand duality. Instead of compact spaces, we use compact groups. They constitute a category KGrp with continuous homomorphisms as maps. Morphisms between compact quantum groups are unital $*$-homomorphisms preserving the comultiplication. They make compact quantum groups into a category KQGrp. As in Gelfand duality, we want to consider the full subcategory CKQGrp of commutative compact quantum groups.

Theorem 3 The functor $C:$ KGrp $^{\text {op }} \rightarrow$ CKQGrp is a dual equivalence between the category of compact Hausdorff groups and commutative compact quantum groups.

If $A$ is a commutative compact quantum group, then the underlying space of its dual group is the spectrum of $A$, considered as $C^{*}$-algebra. The multiplication on $G$ arises from the comultiplication on $A$.

There is another way to assign a compact quantum group to a finite group $G$, namely the group algebra $C[G]$. The elements are again functions from $G$ to $\mathbb{C}$, but now the multiplication is given by convolution:

$$
\varphi * \psi(g)=\sum_{h k=g} \varphi(h) \psi(k)
$$

The standard basis of $C[G]$ consists of Dirac functions $\lambda_{g}$ for $g \in G$, defined by $\lambda_{g}(g)=1$ and $\lambda_{g}(h)=0$ for $h \neq g$. The convolution product assumes a particularly easy form on these basis vectors, namely $\lambda_{g} * \lambda_{h}=\lambda_{g h}$. The comultiplication is defined on basis vectors by $\Delta\left(\lambda_{g}\right)=\lambda_{g} \otimes \lambda_{g}$.

\subsection{Effect Algebras and Modules}

Another duality that we will use involves the effects in a $C^{*}$-algebra. Effects represent probabilistic measurements that can be performed on a physical system. Let $A$ be any $\mathrm{C}^{*}$-algebra. An element $a$ in $A$ is said to be positive if it can be written as $a=b^{*} b$ for some $b \in A$. Positivity can be used to define an order on the self-adjoint part of $A$, called the Löwner order. Let $a, b$ be self-adjoint elements in $A$, then we say that $a \leq b$ if and only if $b-a$ is positive. An effect in $A$ is a self-adjoint $a \in A$ for which $0 \leq a \leq 1$.

Effects in a $C^{*}$-algebra can be organized into an algebraic structure called an effect module. Effect modules were introduced in [4], based on earlier work on effect algebras, which started in [1]. For an overview of the theory about effect algebras, see [2].

Roughly speaking, an effect module looks like a vector space, but the addition is only a partial operation (since the sum of two effects may lie above 1), and we can only multiply by scalars in the unit interval $[0,1]$. Instead of complements with respect to 0 , we have complements with respect to 1 . This means that for every effect $a$ there exists an effect $b$ for which $a+b=1$. The precise definition is as follows.

Definition 4 An effect module consists of a set $A$ equipped with a partial binary operation $\boxplus$ called addition, a unary operation $(-)^{\perp}$ called orthocomplement, a scalar multiplication $\cdot:[0,1] \times A \rightarrow A$ and constants $0,1 \in A$, subject to the following axioms:

- The operation $\boxplus$ is commutative, which means that whenever $a \boxplus b$ is defined, then also $b \boxplus a$ is defined, and $a \boxplus b=b \boxplus a$. 
- The operation $\boxplus$ is associative, which means that if $a \boxplus b$ and ( $a \boxplus b) \boxplus c$ are defined, then also $b \boxplus c$ and $a \boxplus(b \boxplus c)$ are defined, and $(a \boxplus b) \boxplus c=a \boxplus(b \boxplus c)$.

- For every $a \in A, a \boxplus 0=0 \boxplus a=a$.

- For all $a, b \in A, a \boxplus b=1$ if and only if $b=a^{\perp}$.

- If $a \boxplus 1$ is defined, then $a=0$.

- For all $r, s \in[0,1]$ and $a \in A, r \cdot(s \cdot a)=(r s) \cdot a$.

- If $r+s \leq 1$, then $(r+s) \cdot a=r \cdot a+s \cdot a$.

- If $a \boxplus b$ is defined, then $r \cdot(a \boxplus b)=r \cdot a \boxplus r \cdot b$.

- $1 \cdot a=a$.

Effect modules form a category EMod, in which the morphisms are functions preserving addition, orthocomplement, scalar multiplication, and the constants 0 and 1 .

The easiest example of an effect module is the unit interval $[0,1]$. The partial operation is addition, where $a \boxplus b$ is defined if and only if $a+b \leq 1$. The orthocomplement is given by $a^{\perp}=1-a$, and the scalar multiplication is simply the multiplication on $[0,1]$. Another example are the effects in a $C^{*}$-algebra, with the same operations. If $A$ is a $C^{*}$-algebra, then its collection of effects is denoted $\mathcal{E} f(A)$. Any Hilbert space $H$ gives rise to a $\mathrm{C}^{*}$-algebra $\mathcal{B}(H)$, hence to an effect module $\mathcal{E} f(\mathcal{B}(H)$ ). We will often abbreviate this to $\mathcal{E} f(H)$.

More generally, every partially ordered vector space $V$ over $\mathbb{R}$ gives rise to an effect module. Pick an element $u \in V$ for which $u>0$, then the interval $[0, u]=\{v \in V \mid$ $0 \leq v \leq u\}$ is an effect module. Addition serves as the partial binary operation, and the orthocomplement is $v^{\perp}=u-v$. The scalar multiplication is obtained by restricting the scalar multiplication from $\mathbb{R}$ to $[0,1]$. In fact, every effect module is an interval in some partially ordered $\mathbb{R}$-vector space, as shown in [4, Theorem 3.1].

To work with infinite-dimensional vector spaces, it is often necessary to require that they are complete in a certain metric. The same holds for effect modules. If $A$ is an effect module, then a state on $A$ is a morphism $\sigma: A \rightarrow[0,1]$. The collection of all states is written as $\operatorname{St}(A)$. Define a metric on $A$ via

$$
d(a, b)=\sup _{\sigma \in \operatorname{St}(A)}|\sigma(a)-\sigma(b)|
$$

We call the effect module $A$ a Banach effect module if it is complete in its associated metric. Banach effect modules give a full subcategory of EMod written as BEMod.

\subsection{Convex Spaces}

The state space of an effect module is always a compact convex space. We will make this observation more precise by defining a suitable category of compact convex spaces, following [10]. A topological vector space is said to be locally convex if its topology has a base of convex open sets. Let KConv be the category whose objects are compact convex subspaces of a locally convex vector space. A subspace $X \subset V$ is called convex if, for all $x, y \in X$ and $\lambda \in[0,1]$, we have that $\lambda x+(1-\lambda) y \in X$. A morphism between compact convex spaces $X \subset V$ and $Y \subset W$ is a continuous map $f: X \rightarrow Y$ that preserves convex combinations, i.e. $f(\lambda x+(1-\lambda) y)=\lambda f(x)+(1-\lambda) f(y)$. Such a map is called affine.

The state space of an effect module $A$ is contained in the vector space $\{\varphi: A \rightarrow \mathbb{R} \mid$ $\varphi(a \boxplus b)=\varphi(a)+\varphi(b)\}$, which is locally convex. Therefore $\operatorname{St}(A)$ is an object in the category KConv, and St is a contravariant functor from BEMod to KConv. The functor $\operatorname{Hom}_{\mathbf{K C o n v}}(-,[0,1])$ is a contravariant functor in the other direction. The following result is taken from [5, Theorem 6], but see also [10, Section 4]. 
Theorem 5 The functors $\mathrm{St}$ and $\operatorname{Hom}_{\mathbf{K C o n v}}(-,[0,1])$ are inverses of each other. Hence the categories KConv and BEMod are dually equivalent.

Examples 6 We give some examples of convex spaces and their dual effect modules.

(1) If $X$ is a finite set, then let $\mathcal{D}(X)=\left\{f: X \rightarrow[0,1] \mid \sum_{x \in X} f(x)=1\right\}$. This can be visualized as the standard simplex whose vertices are points in $X$. An element $f \in \mathcal{D}(X)$ is usually written as a formal convex combination $\sum_{x \in X} a_{x} x$, where the coefficients are the function values $a_{x}=f(x)$. They are subject to the condition $\sum_{x} a_{x}=1$. This construction gives a functor $\mathcal{D}:$ FinSets $\rightarrow$ KConv, where on a morphism $\varphi: X \rightarrow Y$ we define $\mathcal{D}(\varphi)\left(\sum_{x} a_{x} x\right)=\sum_{x} a_{x} \varphi(x)$. The dual effect module of $\mathcal{D}(X)$ is $\operatorname{Hom}(\mathcal{D}(X),[0,1])$, which is isomorphic to [0, 1] ${ }^{X}$.

(2) In the above example, $\mathcal{D}(X)$ can be thought of as the set of discrete probability measures or distributions on $X$. There is a continuous analogue of this construction. Let $X$ now be a compact Hausdorff space, and let $\Sigma_{X}$ be its Borel $\sigma$-algebra. Denote the space of Radon measures on $X$ by $\mathcal{R}(X)$. A Radon measure is a probability measure $\mu: \Sigma_{X} \rightarrow[0,1]$ that satisfies

$$
\mu(M)=\sup _{\substack{K \subseteq M \\ K \text { compact }}} \mu(K) .
$$

In [3] it is shown that $\mathcal{R}$ forms a monad on the category of compact Hausdorff spaces. Its category of Eilenberg-Moore algebras is equivalent to KConv, so convex spaces of the form $\mathcal{R}(X)$ can be thought of as the free convex spaces over a compact Hausdorff space. The dual effect module of $\mathcal{R}(X)$ is the collection of continuous functions from $X$ into $[0,1]$. This fact is a categorical reformulation of the Riesz-Markov theorem. To see this, observe that there is a map $\mathcal{R}(X) \rightarrow \operatorname{Hom}(C(X,[0,1]),[0,1])$ given by integration, i.e. $\mu \mapsto \int(-) \mathrm{d} \mu$. The Riesz-Markov theorem states that this map is an isomorphism, so $\mathcal{R}(X)$ is the dual of $C(X,[0,1])$. This shows that the following diagram, connecting Gelfand and Kadison duality, commutes:

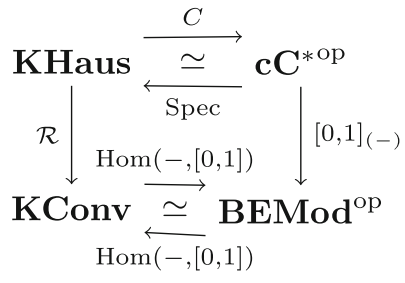

(3) Let $H$ be a Hilbert space. A density matrix on $H$ is a positive trace-class operator $\rho: H \rightarrow H$ with trace 1 . The collection of all density matrices forms a convex space denoted $\mathcal{D} \mathcal{M}(H)$. The importance of this example lies in its connection to the effects on $H$ : there is an isomorphism $\mathcal{E} f(H) \rightarrow \operatorname{Hom}(\mathcal{D} \mathcal{M}(H),[0,1])$, that maps an effect $a$ to the function $\rho \mapsto \operatorname{tr}(\rho a)$. Because this map is an isomorphism, $\mathcal{E} f(H)$ is the dual effect module of $\mathcal{D} \mathcal{M}(H)$.

There are several ways to construct new convex spaces from old ones. In the remainder of this paper we will sometimes use coproducts and tensor products of convex spaces, so we will describe these briefly here. 
The category KConv has all coproducts. The coproduct of two convex spaces can be described geometrically, using the embedding in a locally convex vector space. The following description is a slight modification of the construction in [10]. Suppose that $X \subseteq V$ and $Y \subseteq W$ are compact convex subsets of locally convex vector spaces. Then the coproduct $X+Y$ can be embedded in the vector space $V \oplus W \oplus \mathbb{R}$. To construct this coproduct, embed $X$ in this larger vector space via the inclusion $x \mapsto(x, 0,1)$, and embed $Y$ via the inclusion $y \mapsto(0, y, 0)$. The convex hull of the disjoint union of $X$ and $Y$ is the coproduct of $X$ and $Y$. This is made precise in the following.

Proposition 7 If $X \subseteq V$ and $Y \subseteq W$ are objects in the category KConv, then their coproduct is

$$
X+Y=\{(r x,(1-r) y, r) \mid r \in[0,1], x \in X, y \in Y\} \subseteq V \oplus W \oplus \mathbb{R} .
$$

Proof Define embeddings $i_{X}: X \rightarrow X+Y$ and $i_{Y}: Y \rightarrow X+Y$ via $i_{X}(x)=(x, 0,1)$ and $i_{Y}(y)=(0, y, 0)$. Given affine maps $f: X \rightarrow Z$ and $g: Y \rightarrow Z$, define $h: X+Y \rightarrow Z$ by

$$
h(r x,(1-r) y, r)=r f(x)+(1-r) g(y) .
$$

Then $h \circ i_{X}=f$ and $h \circ i_{Y}=g$, so it remains to be shown that $h$ is the unique map with this property. Suppose that $h^{\prime}: X+Y \rightarrow Z$ is an affine map for which $h \circ i_{X}=f$ and $h \circ i_{Y}=g$. Then

$$
h^{\prime}(r x,(1-r) y, r)=h^{\prime}(r(x, 0,1)+(1-r)(0, y, 0))=r f(x)+(1-r) g(y),
$$

which proves uniqueness.

Example 8 Denote the one-point convex space by 1 . The coproduct $1+\cdots+1$ of $n$ copies of this space is the convex hull of $n$ points, embedded in $\mathbb{R}^{n-1}$ in such a way that they are all affinely independent. Therefore this coproduct is the standard simplex $\mathcal{D}(n)$.

We continue with a discussion of the tensor product of compact convex spaces. If $X, Y$, and $Z$ are compact convex spaces, then a map $X \times Y \rightarrow Z$ is called bi-affine is it is affine in both variables separately. A tensor product of $X$ and $Y$ is a compact convex space $X \otimes Y$ equipped with a bi-affine map $\otimes: X \times Y \rightarrow X \otimes Y$ such that for every compact convex space $Z$ and every bi-affine $f: X \times Y \rightarrow Z$ there exists a unique affine map $g: X \otimes Y \rightarrow Z$ such that $g \circ \otimes=f$. Semadeni proves in [10] that any two compact convex spaces admit a tensor product, and that it is unique up to isomorphism.

The above tensor product enjoys many good properties. The one-point convex space 1 acts as a unit for the tensor. Furthermore, the tensor product distributes over coproducts. From these two facts, together with the isomorphism $\mathcal{D}(n) \cong 1+\cdots+1$, it can be deduced that the tensor product of standard simplices is $\mathcal{D}(n) \otimes \mathcal{D}(m) \cong \mathcal{D}(n m)$.

\section{Kadison Duality for Group and Function Algebras}

Let $G$ be a finite group. This gives rise to two Hopf-algebras, or compact quantum groups, namely the function algebra $C(G)$ and the group algebra $C[G]$. Of these two Hopf-algebras, the function algebra is commutative but in general not cocommutative, while for the group algebra, it is the other way round. Therefore the duality from Theorem 3 only applies to 
the function algebra $C(G)$. However, Kadison duality also applies to unit intervals of noncommutative $\mathrm{C}^{*}$-algebras, so we can use this for both the group algebra and the function algebra.

Definition 9 A convex monoid is an object $X$ of the category KConv, together with a continuous multiplication map $\cdot: X \times X \rightarrow X$ and a constant $1 \in X$, such that

- The operation $\cdot$ is affine in both variables separately, that is, $(\lambda x+(1-\lambda) y) \cdot z=$ $\lambda x \cdot z+(1-\lambda) y \cdot z$ and similarly for convex combinations on the right.

- The operation $\cdot$ is associative.

- 1 is a unit for .

Equivalently, a convex monoid is a convex space $X$ equipped with a map $X \otimes X \rightarrow X$ that is associative and has a unit.

A variant of quantum groups in the framework of Kadison duality should give a duality between effect modules with a comultiplication and convex monoids. In this section we describe these objects for the Hopf-algebras $C(G)$ and $C[G]$. We will start with the function algebra $C(G)$. In fact, this algebra can be defined for any compact group $G$, so we will now determine the effect module and convex space associated to $C(G)$ for an arbitrary compact group $G$.

\section{Proposition 10 Let $G$ be a compact group. Then}

(1) the effect module $\mathcal{E} f(C(G)) \cong\{\varphi \mid \varphi: G \rightarrow[0,1]$ is continuous $\}$. Restriction of $\Delta$ on $\mathcal{E} f(C(G))$ defines a comultiplication map $\mathcal{E} f(C(G)) \rightarrow \mathcal{E} f(C(G \times G))$ (which is a morphism of effect modules);

(2) the state space $\operatorname{St}(\mathcal{E} f(C(G))$ is isomorphic to the space $\mathcal{R}(G)$ of Radon measures on $G$. Moreover, $\mathcal{R}(G)$ is a convex monoid with respect to the multiplication obtained by dualizing $\Delta$ on $\mathcal{E} f(C(G))$.

Proof The effect module $\mathcal{E} f(C(G))$ consists of all functions $\varphi$ for which $0 \leq \varphi \leq 1$. Since the multiplication in $C(G)$ is pointwise, the order is also pointwise, and hence $\mathcal{E} f(C(G))$ consists of continuous maps $G \rightarrow[0,1]$. The comultiplication on $C(G)$ induces a map of effect modules $\Delta: \mathcal{E} f(C(G)) \rightarrow \mathcal{E} f(C(G \times G))$, given by $\Delta(\varphi)(g, h)=\varphi(g h)$. Clearly, $\Delta$ is coassociative.

The state space of $\mathcal{E} f(C(G))$ consists of all morphisms $\sigma: \operatorname{Hom}(G,[0,1]) \rightarrow[0,1]$. By part 2 of Examples 6, this is the same as the space of Radon measures $\mathcal{R}(G)$.

The multiplication on $\mathcal{R}(G)$ can also be decribed directly in terms of the multiplication on $G$. Applying the functor $\mathcal{R}$ to the multiplication map $\cdot: G \times G \rightarrow G$ gives a map $\mathcal{R}(G \times G) \rightarrow \mathcal{R}(G)$. Since $\mathcal{R}(G) \otimes \mathcal{R}(G) \cong \mathcal{R}(G \times G)$, this provides a convex monoid structure on $\mathcal{R}(G)$, which is the dual of $\mathcal{E} f(C(G))$. This convex monoid has been studied categorically in [6].

The multiplication on the group algebra $C[G]$ is more complicated than the one on function algebra. Therefore the Löwner order on $C[G]$ and the effect module are also more difficult to compute explicitly. The algebra $C[G]$ is simultaneously a $\mathrm{C}^{*}$-algebra and a Hilbert space, and the algebra structure is compatible with the inner product, so $C[G]$ forms a Hilbert algebra. We shall use some general facts about Hilbert algebras to compute the effect module and the state space of $C[G]$. 
Definition 11 A Hilbert algebra is a *-algebra $A$ equipped with an inner product $\langle-\mid-\rangle$, such that

(1) For all $a, b \in A,\langle a \mid b\rangle=\left\langle b^{*} \mid a^{*}\right\rangle$.

(2) For each $a \in A$, the map $b \mapsto a b$ is a bounded operator.

(3) For all $a, b, c \in A,\langle a b \mid c\rangle=\left\langle b \mid a^{*} c\right\rangle$.

(4) The linear span of $\{a b \mid a, b \in A\}$ is dense in $A$.

For more about Hilbert algebras see [7]. We will mainly work with unital Hilbert algebras, in which the fourth property holds automatically.

Lemma 12 Let $A$ be a Hilbert algebra, and $f: A \rightarrow$ A a map of left A-actions, i.e. a map satisfying $f(a b)=a f(b)$. Then:

(1) If $f$ is positive, then $\sqrt{f}$ is also a map of left A-actions.

(2) The adjoint $f^{\dagger}$ is a map of left A-actions.

(3) $f^{\dagger}(1)=f(1)^{*}$.

Proof

(1) The square root $\sqrt{f}$ commutes with every operator that commutes with $f$.

(2) It suffices to prove that $f^{\dagger}(a b)$ and $a f^{\dagger}(b)$ have the same inner product with any $x \in A$. This holds because

$$
\begin{aligned}
\left\langle f^{\dagger}(a b) \mid x\right\rangle=\langle a b \mid f(x)\rangle=\left\langle b \mid a^{*} f(x)\right\rangle=\left\langle b \mid f\left(a^{*} x\right)\right\rangle & =\left\langle f^{\dagger}(b) \mid a^{*} x\right\rangle \\
& =\left\langle a f^{\dagger}(b) \mid x\right\rangle .
\end{aligned}
$$

$$
\left\langle f(1)^{*} \mid x\right\rangle=\left\langle x^{*} \mid f(1)\right\rangle=\langle 1 \mid x f(1)\rangle=\langle 1 \mid f(x)\rangle=\left\langle f^{\dagger}(1) \mid x\right\rangle .
$$

Since any Hilbert algebra $A$ is a *-algebra, it can be ordered, and hence we can speak about effects in the algebra. These are elements $a \in A$ such that $0 \leq a \leq 1$. But $A$ is also a Hilbert space, so we can also speak about effects on the Hilbert algebra, which are maps $\varepsilon: A \rightarrow A$ that lie between 0 and $\operatorname{id}_{A}$. The next result connects effects in $A$ with effects on $A$.

Proposition 13 Let $A$ be a unital Hilbert algebra. There is a bijective correspondence between:

(1) Effects in A, i.e. $a \in A$ for which $0 \leq a \leq 1$;

(2) Effects $\varepsilon: A \rightarrow A$ that are also maps of left A-actions.

Proof If $\varepsilon: A \rightarrow A$ is an effect for which $\varepsilon(a b)=a \varepsilon(b)$, then $\varepsilon(1)$ is an effect in $A$. To show this, we will start by proving positivity. The effect $\varepsilon$ has a positive square root $\sqrt{\varepsilon}$. We claim that $\sqrt{\varepsilon}(1) \sqrt{\varepsilon}(1)=\varepsilon(1)$. This follows from the following computation, using Lemma 12:

$$
\begin{aligned}
\langle\varepsilon(1) \mid x\rangle & =\langle\sqrt{\varepsilon} \sqrt{\varepsilon}(1) \mid x\rangle=\left\langle\sqrt{\varepsilon}(1) \mid \sqrt{\varepsilon}^{\dagger}(x)\right\rangle \\
& =\left\langle\sqrt{\varepsilon}(1) \mid x \sqrt{\varepsilon}^{\dagger}(1)\right\rangle=\left\langle\sqrt{\varepsilon}(1) \mid x \sqrt{\varepsilon}(1)^{*}\right\rangle \\
& =\left\langle\sqrt{\varepsilon}(1) x^{*} \mid \sqrt{\varepsilon}(1)^{*}\right\rangle=\left\langle x^{*} \mid \sqrt{\varepsilon}(1)^{*} \sqrt{\varepsilon}(1)^{*}\right\rangle=\langle\sqrt{\varepsilon}(1) \sqrt{\varepsilon}(1) \mid x\rangle .
\end{aligned}
$$

This shows that $\varepsilon(1)$ has a square root, so it is positive. Similarly, since the square root $\sqrt{I-\varepsilon}$ exists, the element $1-\varepsilon(1) \in A$ is positive. Therefore $\varepsilon(1)$ is an effect. 
Conversely, if $a \in A$ is an effect, define $\varepsilon: A \rightarrow A$ by $\varepsilon(x)=x a$. Then $\varepsilon$ is clearly a map of left actions. Since $a$ is positive, there is a $b$ such that $a=b^{*} b$. Define $\beta: A \rightarrow A$ by $\beta(x)=x b$. Then $\beta \beta^{\dagger}(x)=x b^{*} b=\varepsilon(x)$, so $\varepsilon$ is positive. Analogously we can prove $\varepsilon \leq I$, hence $\varepsilon$ is an effect. It is easy to see that both constructions are mutually inverse.

Proposition 14 Let $V$ be a unitary representation of $G$. Write the decomposition of $V$ into irreducible representations as $V=n_{1} V_{1} \oplus \cdots \oplus n_{k} V_{k}$. Then the effect module $\{\varepsilon: V \rightarrow$ $V \mid \varepsilon$ is effect and intertwiner $\}$ is isomorphic to $\mathcal{E} f\left(\mathbb{C}^{n_{1}}\right) \times \cdots \times \mathcal{E} f\left(\mathbb{C}^{n_{k}}\right)$.

Proof An intertwining effect $\varepsilon: V \rightarrow V$ can be written as a matrix of maps $\varepsilon_{i j}: n_{i} V_{i} \rightarrow$ $n_{j} V_{j}$. By Schur's Lemma, each $\varepsilon_{i j}=0$ for $i \neq j$. The effects $\varepsilon_{i i}$ can in turn be decomposed into an $n_{i} \times n_{i}$ matrix of maps $V_{i} \rightarrow V_{i}$, and these are all scalar multiples of the identity by Schur's Lemma. Therefore each $\varepsilon_{i i}$ corresponds to an effect on $\mathbb{C}^{n_{i}}$.

Theorem 15 Let $G$ be a finite group, and let $V_{1}, \ldots, V_{k}$ be its irreducible representations. Then

(1) the effect module $\mathcal{E} f(C[G])$ is isomorphic to $\mathcal{E} f\left(V_{1}\right) \times \cdots \times \mathcal{E} f\left(V_{k}\right)$. The comultiplication map $\hat{\Delta}: \mathcal{E} f(C[G]) \rightarrow \mathcal{E} f(C[G \times G])$ given by $\hat{\Delta}\left(\sum_{g} a_{g} \lambda_{g}\right)=\sum_{g} a_{g} \lambda_{(g, g)}$;

(2) The state space of $C[G]$, denoted by $\operatorname{St}(C[G])$, is the coproduct $\mathcal{D} \mathcal{M}\left(V_{1}\right)+\cdots+$ $\mathcal{D M}\left(V_{k}\right)$ in the category of convex spaces. Moreover, $\operatorname{St}(C[G])$ is a convex monoid with respect to the multiplication $\mu: \operatorname{St}(C[G \times G]) \rightarrow \operatorname{St}(C[G])$ defined as the linear extension of $\mu(\sigma)\left(\lambda_{g}\right)=\sigma\left(\lambda_{(g, g)}\right)$.

Proof By Proposition 13, the effect module $\mathcal{E} f(C[G])$ is isomorphic to $\{\varepsilon: C[G] \rightarrow$ $C[G] \mid \varepsilon$ is effect and map of left $C[G]$-actions $\}$. The condition that $\varepsilon$ is a map of left $C[G]-$ actions means that it is an intertwiner from the regular $G$-representation $C[G]$ to itself. The regular representation decomposes as $C[G]=n_{1} V_{1} \oplus \cdots \oplus n_{k} V_{k}$, so by Proposition 14 $\mathcal{E} f(C[G])$ is isomorphic to $\mathcal{E} f\left(\mathbb{C}^{\operatorname{dim} V_{1}}\right) \times \cdots \times \mathcal{E} f\left(\mathbb{C}^{\operatorname{dim} V_{k}}\right) \cong \mathcal{E} f\left(V_{1}\right) \times \cdots \times \mathcal{E} f\left(V_{k}\right)$. This is a Banach effect module, so we can use the duality between convex compact spaces and Banach effect modules to determine the dual space. Dualizing turns products into coproducts, so the dual space is $\mathcal{D} \mathcal{M}\left(V_{1}\right)+\cdots+\mathcal{D} \mathcal{M}\left(V_{k}\right)$. Clearly, $\hat{\Delta}$ is coassociative.

Dualizing $\hat{\Delta}$ gives $\mu$ on the state space $\operatorname{St}(C[G])$. The convex monoid structure on the state space of the group algebra satisfies $(\sigma \cdot \tau)\left(\lambda_{g}\right)=\sigma\left(\lambda_{g}\right) \tau\left(\lambda_{g}\right)$ on basis vectors.

\section{Convex Pontryagin Duality for Group and Function Algebras}

The group algebra and the function algebra associated to a finite group are both finitedimensional Hopf algebras. These are related via a non-commutative generalization of Pontryagin duality, see e.g. [8] for details. In the previous section, we found two convex monoids that can be obtained from a finite group: the state space $\mathcal{D}(G)$ of the function algebra, and the state space $\mathcal{D} \mathcal{M}\left(V_{1}\right)+\cdots+\mathcal{D} \mathcal{M}\left(V_{k}\right)$ of the group algebra, where the $V_{i}$ are the irreducible representations of $G$. This section will present a construction to convert these two convex monoids into each other. This construction can be viewed as a convex counterpart of Pontryagin duality.

Definition 16 A linear representation of a convex monoid $X$ consists of a vector space $V$ and a monoid homomorphism $\rho: X \rightarrow \operatorname{End}(V)$ that preserves convex combinations. 
As usual, a representation can also be written as an action of $X$ on $V$, that is, a map $X \times V \rightarrow V$. A linear representation of a convex monoid is then required to be affine in the first variable and linear in the second variable. We will look at the linear representations of the convex monoid $\mathcal{D}(G)$.

Lemma 17 There is a one-to-one correspondence between representations of the finite group $G$ and linear representations of $\mathcal{D}(G)$.

Proof Representations of $G$ are monoid homomorphisms $G \rightarrow \operatorname{End}(V)$, since all monoid homomorphisms between groups are automatically group homomorphisms. Linear representations of $\mathcal{D}(G)$ are monoid homomorphisms $\mathcal{D}(G) \rightarrow \operatorname{End}(V)$ that are also morphisms of convex spaces. Since $\mathcal{D}(G)$ is the free convex space generated by $G$, it follows that there is a one-to-one correspondence between maps of sets $G \rightarrow \operatorname{End}(V)$ and maps of convex spaces $\mathcal{D}(G) \rightarrow \operatorname{End}(V)$. It is easy to check that this equivalence restricts to monoid homomorphisms.

This result produces an easy way to construct the state space of $C[G]$ out of the state space of $C(G)$, in the following steps:

(1) Let $V_{1}, \ldots, V_{k}$ be the irreducible linear representations of $\operatorname{St}(C(G))$.

(2) Form the convex sets of density matrices $\mathcal{D} \mathcal{M}\left(V_{i}\right)$ for each $i$.

(3) The coproduct (in the category $\mathbf{K C o n v}$ ) of all $\mathcal{D} \mathcal{M}\left(V_{i}\right)$ is the state space of $C[G]$.

Since irreducible representations of $G$ are the same as irreducible linear representations of $\mathcal{D}(G)$, this construction yields exactly the state space of $C[G]$. A surprising fact is that it works in two directions: if we apply exactly the same construction to the state space of $C[G]$, we end up with the state space of $C(G)$.

Proposition 18 Let $V_{1}, \ldots, V_{k}$ be the irreducible linear representations of the convex monoid $\operatorname{St}(C[G])$. Then the convex space $\mathcal{D} \mathcal{M}\left(V_{1}\right)+\cdots+\mathcal{D} \mathcal{M}\left(V_{k}\right)$ is isomorphic to $\operatorname{St}(C(G))$.

Proof We will determine the irreducible representations $V_{i}$. Recall that the multiplication on $\operatorname{St}(C[G])$ was given by $\sigma \cdot \tau\left(\lambda_{g}\right)=\sigma\left(\lambda_{g}\right) \tau\left(\lambda_{g}\right)$. Therefore this convex monoid is commutative. All irreducible representations of a commutative monoid are 1-dimensional. Each $g \in G$ gives a 1-dimensional linear representation $\rho_{g}: \operatorname{St}(C[G]) \rightarrow \mathbb{C}$ by $\rho_{g}(\sigma)=\sigma(g)$.

We will now check that all 1-dimensional linear representations are of the form $\rho_{g}$ for some $g \in G$. Let $\rho: \operatorname{St}(C[G]) \rightarrow \mathbb{C}$ be an arbitrary representation. Then the map $\rho$ extends to a function $\operatorname{Hom}(C[G], \mathbb{C}) \rightarrow \mathbb{C}$ in the double dual of $C[G]$, hence there exists $a \in C[G]$ such that $\sigma(a)=\rho(\sigma)$ for all states $\sigma$ on $C[G]$. We will show that $a$ is actually an element in $G \subseteq C[G]$. Express $a$ as $a=a_{1} \lambda_{g_{1}}+\cdots+a_{n} \lambda_{g_{n}}$. Then, for any two states $\sigma$ and $\tau$,

$$
\rho(\sigma \tau)=\sigma \tau\left(\sum_{i} a_{i} \lambda_{g_{i}}\right)=\sum_{i} a_{i} \sigma\left(\lambda_{g_{i}}\right) \tau\left(\lambda_{g_{i}}\right)
$$

and

$$
\rho(\sigma) \rho(\tau)=\sigma\left(\sum_{i} a_{i} \lambda_{g_{i}}\right) \tau\left(\sum_{j} a_{j} \lambda_{g_{j}}\right)=\sum_{i, j} a_{i} a_{j} \sigma\left(\lambda_{g_{i}}\right) \tau\left(\lambda_{g_{j}}\right) .
$$

The map $\rho$ is a representation, so these two expressions must be equal for all states $\sigma$ and $\tau$. Comparing coefficients shows that at most one $a_{i}$ is equal to 1 , and all others are 0 . The element $a$ cannot be identically 0 , since $\rho$ preserves 1 . Hence $a$ is equal to $\lambda_{g}$ for some $g \in G$, which proves that the maps $\rho_{g}$ are indeed the only 1-dimensional representations. 
There is only one density matrix on any 1-dimensional space. Therefore the space $\mathcal{D} \mathcal{M}\left(V_{1}\right)+\cdots+\mathcal{D} \mathcal{M}\left(V_{k}\right)$ is a coproduct of \#G copies of the one-point space, which is $\mathcal{D}(G)$.

We have shown that if we start with the convex monoid $\operatorname{St}(C(G)) \cong \mathcal{D}(G)$ and apply the above construction twice, then we get back a convex space that is isomorphic to the underlying space of the original convex monoid. Now we wish to show that the multiplication is also preserved in this construction, so that we obtain an isomorphism of convex monoids, rather than just convex spaces. For this we have to endow the coproduct of density matrices with a multiplication. It is useful to have an explicit isomorphism between $\mathcal{D} \mathcal{M}\left(V_{1}\right)+\cdots+\mathcal{D} \mathcal{M}\left(V_{k}\right)$ and $\operatorname{St}(C[G])$.

Lemma 19 Let $\left(V_{1}, \rho_{1}\right), \ldots,\left(V_{k}, \rho_{k}\right)$ be the irreducible representations of $G$. The map $\Phi: \mathcal{D M}\left(V_{1}\right)+\cdots+\mathcal{D} \mathcal{M}\left(V_{k}\right) \rightarrow \operatorname{St}(C[G])$ determined by $\Phi(T)\left(\lambda_{g}\right)=\operatorname{tr}\left(T \rho_{i}(g)\right)$ for $T \in \mathcal{D} \mathcal{M}\left(V_{i}\right)$ is an isomorphism of convex spaces.

Proof Consider the map $\Psi: C[G] \rightarrow \operatorname{End}\left(V_{1}\right) \times \cdots \times \operatorname{End}\left(V_{k}\right)$ of $C^{*}$-algebras, on basis vectors determined by $\lambda_{g} \mapsto\left(\rho_{1}(g), \ldots, \rho_{k}(g)\right)$. We claim that this map is injective. Suppose that $a, b \in C[G]$ are such that $\rho_{i}(a)=\rho_{i}(b)$ for all $i$. Then $a$ and $b$ act in the same way in all irreducible representations of $G$. Since any representation of $G$ can be decomposed into irreducibles, $a$ and $b$ act in the same way in all representations of $G$. In particular, they have the same action on the regular representation $C[G]$. Thus $a=a \cdot e=b \cdot e=b$. Since $\Psi$ is injective and its domain has the same dimension as its codomain, it is an isomorphism.

Taking states of a $\mathrm{C}^{*}$-algebra provides a contravariant functor $\mathrm{St}: \mathbf{C}^{*} \rightarrow \mathbf{K C o n v}$. Therefore, applying the state functor to $\Psi$ gives a map $\operatorname{St}\left(\operatorname{End}\left(V_{1}\right)\right)+\cdots+\operatorname{St}\left(\operatorname{End}\left(V_{k}\right)\right) \rightarrow$ $\operatorname{St}(C[G])$. There is an isomorphism $\alpha: \mathcal{D} \mathcal{M}\left(V_{i}\right) \rightarrow \operatorname{St}\left(\operatorname{End}\left(V_{i}\right)\right)$ given by $\alpha(\rho)(A)=$ $\operatorname{tr}(\rho A)$, and hence $\operatorname{St}(\Psi)=\Phi$. Since $\Psi$ is an isomorphism and $\mathrm{St}$ is a functor, $\Phi$ is also an isomorphism.

Using this isomorphism, the multiplication on the coproduct of density matrices can be described explicitly. Since we are working in a coproduct, it suffices to describe $T \cdot S$, where $T \in \mathcal{D} \mathcal{M}\left(V_{i}\right)$ and $S \in \mathcal{D} \mathcal{M}\left(V_{j}\right)$. Applying the isomorphism $\Phi$ from the lemma above gives states $\lambda_{g} \mapsto \operatorname{tr}\left(T \rho_{i}(g)\right)$ and $\lambda_{g} \mapsto \operatorname{tr}\left(S \rho_{j}(g)\right)$ on $\mathbb{C}[G]$. Multiplying these states pointwise and using properties of the trace gives the map $\lambda_{g} \mapsto \operatorname{tr}\left((T \otimes S)\left(\rho_{i} \otimes \rho_{j}\right)(g)\right)$. Since $\Phi$ is an isomorphism, there is a unique $\sum_{i} \lambda_{i} T_{i} \in \mathcal{D} \mathcal{M}\left(V_{1}\right)+\cdots+\mathcal{D} \mathcal{M}\left(V_{k}\right)$ for which

$$
\sum_{i} \lambda_{i} \operatorname{tr}\left(T_{i} \rho_{i}(g)\right)=\operatorname{tr}\left((T \otimes S)\left(\rho_{i} \otimes \rho_{j}\right)(g)\right) .
$$

We define $T \cdot S$ to be this convex combination $\sum_{i} \lambda_{i} T_{i}$. With the proposition and lemma above, we have now proven the following result.

Theorem 20 Let $G$ be a finite group, and let $V_{1}, \ldots, V_{k}$ be the irreducible linear representations of the convex monoid $\operatorname{St}(C(G))$. Then the convex monoid $\mathcal{D} \mathcal{M}\left(V_{1}\right)+\cdots+\mathcal{D} \mathcal{M}\left(V_{k}\right)$ with multiplication described above is isomorphic to the convex monoid $\operatorname{St}(C[G])$ with pointwise multiplication. 


\section{Convex Pontryagin Duality for a Tensor Product}

The category of finite-dimensional Hopf algebras is self-dual. The dual of a finitedimensional Hopf algebra $A$ is $\hat{A}=\{f: A \rightarrow \mathbb{C} \mid f$ linear $\}$. Its multiplication is derived from the comultiplication on $A$, and vice versa. The Hopf algebras $C(G)$ and $C[G]$ coming from a finite group $G$ are duals of each other via this construction.

Let $A$ be either $C(G)$ or $C[G]$. The main result from the previous section states that if $V_{1}, \ldots, V_{k}$ are the irreducible representations of the convex monoid $\operatorname{St}(A)$, then $\mathcal{D} \mathcal{M}\left(V_{1}\right)+$ $\cdots+\mathcal{D} \mathcal{M}\left(V_{k}\right)$ is isomorphic to $\operatorname{St}(\hat{A})$. This raises the question if this holds for all Hopf algebras. We do not yet know if this is the case in general, but we will now discuss another example of a Hopf algebra for which it holds, so this may be promising for the general case.

Let $G$ be a finite group. Consider the Hopf algebra $A=C(G) \otimes C[G]$, i.e. the tensor product of the function algebra and the group algebra. This Hopf algebra is neither commutative nor cocommutative. Since dualizing preserves tensor products, the dual of $A$ is isomorphic to $A$ itself. Thus the statement that connects the state space of $A$ to its dual amounts to the following.

Proposition 21 Let $V_{1}, \ldots, V_{k}$ be the irreducible representations of the convex monoid $\operatorname{St}(C(G) \otimes C[G])$. Then $\mathcal{D} \mathcal{M}\left(V_{1}\right)+\cdots+\mathcal{D} \mathcal{M}\left(V_{k}\right)$ is isomorphic to $\operatorname{St}(C(G) \otimes C[G])$.

Proof We will first show that $\operatorname{St}(C(G) \otimes C[G])$ is isomorphic to $\operatorname{St}(C(G)) \otimes \operatorname{St}(C[G])$. Since the $C^{*}$-algebra $C(G)$ is commutative and finite-dimensional, it is isomorphic to $\mathbb{C}^{n}$ for some $n$. Hence we have

$$
\operatorname{St}(C(G) \otimes C[G]) \cong \operatorname{St}\left(\mathbb{C}^{n} \otimes C[G]\right) \cong \operatorname{St}\left(C[G]^{\oplus n}\right) .
$$

The state space of a direct sum is the coproduct of state spaces, so this is isomorphic to

$$
\begin{aligned}
\operatorname{St}(C[G])+\cdots+\operatorname{St}(C[G]) & \cong(1+\cdots+1) \otimes \operatorname{St}(C[G]) \\
& \cong \mathcal{D}(n) \otimes \operatorname{St}(C[G]) \\
& \cong \operatorname{St}(C(G)) \otimes \operatorname{St}(C[G])
\end{aligned}
$$

The irreducible representations of a tensor product are tensor products of irreducible representations. The irreducible representations of $\operatorname{St}(C(G))$ are precisely those of $G$; call these $W_{1}, \ldots, W_{m}$. There are $\# G=n$ irreducible representations of $C[G]$ and these are all one-dimensional. Denote these by $W_{1}^{\prime}, \ldots, W_{n}^{\prime}$. Then the irreducible representations of $\operatorname{St}(C(G) \otimes C[G])$ are the tensor products $W_{i} \otimes W_{j}^{\prime}$. Therefore the sum of density matrices is

$$
\begin{aligned}
\sum_{i, j} \mathcal{D} \mathcal{M}\left(W_{i} \otimes W_{j}^{\prime}\right) & \cong\left(\sum_{i} \mathcal{D} \mathcal{M}\left(W_{i}\right)\right)^{+n} \\
& \cong(1+\cdots+1) \otimes \sum_{i} \mathcal{D} \mathcal{M}\left(W_{i}\right) \\
& \cong \mathcal{D}(G) \otimes \sum_{i} \mathcal{D} \mathcal{M}\left(W_{i}\right) \\
& \cong \operatorname{St}(C(G)) \otimes \operatorname{St}(C[G]) \\
& \cong \operatorname{St}(C(G) \otimes C[G])
\end{aligned}
$$

which is what we wanted to show. 
Acknowledgments We would like to thank Johan Commelin, Robert Furber, Philip Scott and Thomas Timmermann for helpful discussions. The first author has been financially supported by the Netherlands Organisation for Scientific Research (NWO) under TOP-GO grant no. 613.001.013 (The logic of composite quantum systems). The second author has been supported by Fields-Ontario postdoctoral fellowship, NSERC and ERA at the University of Ottawa and Carleton University.

Open Access This article is distributed under the terms of the Creative Commons Attribution 4.0 International License (http://creativecommons.org/licenses/by/4.0/), which permits unrestricted use, distribution, and reproduction in any medium, provided you give appropriate credit to the original author(s) and the source, provide a link to the Creative Commons license, and indicate if changes were made.

\section{References}

1. Bennett, M.K., Foulis, D.J.: Effect algebras and unsharp quantum logics. Found. Phys. 24(10), 13311352 (1994). doi:10.1007/BF02283036. MR 1304942

2. Dvurečenskij, A., Pulmannová, S.: New trends in quantum structures, Mathematics and its Applications, vol. 516. Kluwer Academic Publishers, Dordrecht; Ister Science, Bratislava (2000). MR 1861369

3. Furber, R., Jacobs, B.: From Kleisli categories to commutative C*-algebras: probabilistic Gelfand duality. Logical methods in computer science 11(2:5), 1-28 (2015). doi:10.2168/LMCS-11(2:5)2015

4. Gudder, S.P., Pulmannová, S.: Representation theorem for convex effect algebras. Comment. Math. Univ. Carolin. 39(4), 645-659 (1998). MR 1715455

5. Jacobs, B., Mandemaker, J.: The Expectation Monad in Quantum Foundations, eprint. arXiv:1112.3805v2 (2012)

6. Mislove, M.: Probabilistic Monads, Domains and Classical Information, eprint. arXiv:1207.7150v1 (2012)

7. Takesaki, M.: Theory of operator algebras. II, Encyclopaedia of Mathematical Sciences, vol. 125. Springer, Berlin (2003). Operator Algebras and Non-commutative Geometry, 6. MR 1943006

8. Timmermann, T.: An invitation to quantum groups and duality. European Mathematical Society (EMS), Zürich (2008). MR 2397671

9. Woronowicz, S.L.: Compact matrix pseudogroups. Comm. Math. Phys. 111(4), 613-665 (1987) available at http://projecteuclid.org/euclid.cmp/1104159726. MR 901157

10. Semadeni, Z.: Categorical methods in convexity. In: Proceedings of the Colloquium on Convexity, pp. 281-307 (1965) 